la revue La revue pour l'histoire du CNRS

POUR L'HISTOIRE DU CNRS 7 /2002

La biologie | Menaces sur les sciences sociales vers

1980

\title{
Créations et fonctions des stations maritimes
}

françaises

Jean-Louis Fischer

\section{OpenEdition}

Journals

Édition électronique

URL : https://journals.openedition.org/histoire-cnrs/537

DOI : 10.4000/histoire-cnrs. 537

ISSN : 1955-2408

Éditeur

CNRS Éditions

Édition imprimée

Date de publication : 5 novembre 2002

ISBN : 978-2-271-06067-9

ISSN : 1298-9800

Référence électronique

Jean-Louis Fischer, "Créations et fonctions des stations maritimes françaises », La revue pour I'histoire du CNRS [En ligne], 7| 2002, mis en ligne le 17 octobre 2006, consulté le 20 mai 2021. URL : http://journals.openedition.org/histoire-cnrs/537 ; DOI : https://doi.org/10.4000/histoire-cnrs.537

Ce document a été généré automatiquement le 20 mai 2021.

Comité pour l'histoire du CNRS 


\title{
Créations et fonctions des stations maritimes françaises
}

\author{
Jean-Louis Fischer
}

Voyage au bord de la mer

1 L'intérêt croissant que portèrent les naturalistes, à partir des dernières décennies $d u$ XVIII ${ }^{\mathrm{e}}$ siècle, à l'étude de la faune et de la flore marines les conduisit, dans un premier temps, à organiser des voyages sur les lieux mêmes de leurs recherches. Que des naturalistes comme Victor Audouin (1797-1841) et Henri Milne-Edwards (1800-1885) effectuent à plusieurs reprises pendant les années 1826 à 1829 des expéditions sur les côtes de Normandie (région de Granville, îles de Chausey, Saint-Malo, cap Fréhel) est significatif de cette volonté de mettre en place les nouvelles disciplines naturalistes qui ont pour objet le monde de la mer, encore peu connu et plein de promesses de découvertes et de nouveautés.

2 Au début des études de biologie marine, les naturalistes ne possédaient pas de laboratoire et l'auberge fut leur premier lieu d'accueil pour effectuer leurs travaux scientifiques. Ensuite, le marché, l'étal des poissonniers et les discussions avec les pêcheurs représentèrent des sources précieuses de renseignements et d'approvisionnements pour le naturaliste. Carl Vogt (1817-1895), qui défend l'idée de fonder des stations maritimes ${ }^{1}$ dès le début des années 1850 , ne tarit pas d'éloges lors de ses inspections des pêches proposées aux chalands du marché de Nice. Il évoque son étonnement devant la richesse et les variétés de la faune pélagique de la baie de Villefranche ${ }^{2}$. Le naturaliste a compris qu'il est nécessaire « d'étudier la nature dans la nature même et non plus seulement sur les animaux défigurés des collections ${ }^{3}$ ». Pour effectuer ses travaux, le naturaliste explorateur s'installait dans une auberge ou un cabaret situé près du lieu de ses investigations : l'auberge devenait le premier laboratoire improvisé de biologie marine et la même table sert souvent à la fois de paillasse pour ses observations et dissections et pour prendre ses repas. Cette situation ne fut pas toujours très favorable à la réputation de ces nouveaux observateurs de la mer qui devinrent parfois les "bêtes noires " de certains aubergistes en raison du 
désordre qu'ils apportaient dans des lieux initialement tranquilles destinés au repos du voyageur.

3 C'est ainsi, dans ces conditions particulières et pittoresques, que les " naturalistes abordèrent la mer, ses richesses, ses problèmes, bien avant que les peintres en aient vu les couleurs, les écrivains la poésie, et que les mondains en aient décrété le charme ${ }^{4}$ ».

La fondation des stations maritimes

4 La fondation des stations maritimes répond à deux objectifs : d'une part, la nécessité du développement d'une nouvelle direction à donner aux recherches fondamentales en biologie ; d'autre part, une recherche appliquée visant l'exploitation des ressources de la mer. A. de Quatrefages ${ }^{5}$ (1810-1892) insistait sur la complémentarité en zoologie entre la recherche fondamentale et la « pratique » : il évoquait le rôle de l'insémination artificielle (recherche fondamentale) dans la production de poissons pour la consommation (application dans un but économique). Voyageur naturaliste sur les côtes de France et de Sicile, il se familiarise avec la faune marine et milite pour que la zoologie dépasse ses seules ambitions systématiques en s'ouvrant à la " méthode » et à la physiologie. C'est dans une direction comparable que H. Lacaze-Duthiers (1821-1901) promulgue en 1872 une "zoologie expérimentale " s'opposant ainsi au concept bernardien qui considérait la zoologie comme science uniquement contemplative car seule, pour lui, la physiologie pouvait être qualifiée d'expérimentale.

Quoi qu'il en soit, le monde de la mer interpellait les spécialistes des sciences de la vie. Aussi ont-ils conçu l'urgence de créer des structures solides pour accueillir les scientifiques intéressés par ce nouveau monde des eaux salées, saumâtres voire douces, car si ces nouvelles institutions - les stations maritimes - devaient regarder essentiellement la mer, elles ne devaient pas pour autant négliger les côtes et les terres de leur entourage immédiat.

6 Nous ne saurions négliger dans cette approche de la fondation des stations maritimes l'impact de la pensée de Charles Darwin (1809-1882) ni celle de Ernst Haeckel (1834-1919) sur l'impérative nécessité de rechercher nos origines dans le milieu marin, C. Darwin avec L'Origine des espèces (1859) et le concept de « sélection naturelle » et E. Haeckel avec sa Generelle Morphologie des Organismen (1866) et la formulation de sa loi de biogénétique fondamentale. D'où l'importance des recherches concernant les filiations spécifiques des organismes marins et les nombreuses monographies qui en découlent, fondées sur des recherches morphologiques, histologiques, embryologiques (et plus tard moléculaires). Mais la zoologie et l'embryologie descriptives, comparées et évolutives, développées dans les stations maritimes ont été quelque peu supplantées dans les années 1885-1895 par une physiologie et une embryologie expérimentales. Des stations se sont distinguées dans ces spécialités : celle de Tamaris avec Raphaël Dubois et sa découverte du mécanisme de la bioluminescence (1895) ; celle de Roscoff avec Yves Delage et la parthénogenèse expérimentale (1900-1910), pour ne citer que ces deux exemples.

7 Les côtes françaises s'échelonnent dans une variété de paysages sur environ $3000 \mathrm{~km}$. $\mathrm{Du}$ nord à l'ouest et au sud, la faune et la flore sont remarquablement diversifiées et

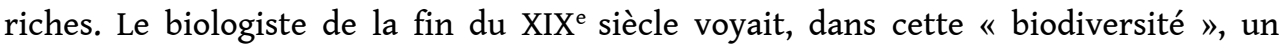
matériel précieux pour élargir les connaissances sur notre environnement en particulier et sur la vie en général. La vie marine a de quoi étonner ses premiers observateurs : Audouin et Edwards (1826-1829) ont fondé la bionomie en décrivant la distribution topographique des invertébrés marins dans leur répartition horizontale et 
verticale. La vie dans le milieu marin est estimée comme possible en 1850 à une profondeur d'environ 600 à $800 \mathrm{~m}$; puis, en 1859, lors d'une réparation du câble télégraphique qui relie la France à l'Algérie, on découvre des animaux fixés sur ce câble immergé à $2000 \mathrm{~m}$ (la surprise des biologistes concernant la vie possible à des grandes profondeurs ne s'arrêtera pas là : en 1977, on découvre, à proximité des sources hydrothermales, des écosystèmes composés de vertébrés, invertébrés et microorganismes, des extrêmophiles, à des profondeurs de $10000 \mathrm{~m}$ ).

Les stations maritimes françaises

Les enjeux scientifiques de l'étude du matériel marin ont stimulé les initiatives et, en moins de trente ans (1872-1899), on a vu fleurir sur les côtes françaises une douzaine de stations maritimes ${ }^{6}$, avec deux exceptions pour la plus ancienne et la plus récente si nous restons dans l'histoire de la fin du xixe siècle : Concarneau fondée en 1859 par Victor Coste et Monaco en 1910.

9 Concarneau est l'exemple de la station qui s'ouvre à une recherche pratique avec la création de grands viviers $(50 \mathrm{~m}$ sur $30 \mathrm{~m})$ pour tester les possibilités d'élevage d'huîtres, de crustacés (homards) et de poissons pour la consommation. Cette pratique de l'élevage, voire de la reproduction en milieu fermé qui s'ouvre vers une économie de marché, fut la pierre de touche qui permit à V. Coste d'obtenir, auprès de Napoléon III, d'importants fonds pour la construction et le fonctionnement de ce premier « vivierlaboratoire ». Ce premier centre de la mer est inspiré par l'organisation de grands centres de pisciculture, comme celui de Huningue (Haut-Rhin, créé en 1852) présidé par V. Coste et celui de Comacchio en Italie, alors célèbre pour ses élevages d'anguilles. Derrière cette idée, il y a celle du développement d'une recherche fondamentale qui fera de Concarneau l'un des premiers lieux de la naissance de l'embryologie expérimentale, discipline scientifique fondamentale qui va changer notre façon d'appréhender le développement embryonnaire par l'analyse du devenir des premières cellules (blastomères) de l'embryon-zygote : c'est Laurent Chabry (1855-1893) qui réalise à Concarneau cette approche expérimentale de l'embryon.

Inauguré les 29 mars et $1^{\text {er }}$ avril 1910, le Musée océanographique de Monaco est construit pour abriter, conserver et montrer non seulement les collections que ramenait le prince Albert Ier de Monaco de ses campagnes scientifiques débutées dès 1885, mais aussi des instruments, du matériel servant aux récoltes, à la pêche, à la navigation. Aux collections de vertébrés et d'invertébrés marins s'ajoute un aquarium public. Ainsi le Musée océanographique de Monaco, est, en 1910, une vitrine généraliste du monde de la mer, destinée d'une part aux scientifiques qui désirent travailler sur les collections (agrémentées d'une bibliothèque) et, d'autre part, au public, pour le " rendre [...] sensible au rôle joué par la mer tant dans l'art, l'ethnographie ou l'économie que dans les connaissances scientifiques ${ }^{7}$ ». Au Musée océanographique s'ajoute l'Institut océanographique situé à Paris, inauguré le 23 janvier 1911 et destiné à l'enseignement et à la recherche expérimentale.

Une station maritime modèle à Naples

$11 \mathrm{Si}$, pendant les trente dernières années du XIX siècle, l'effort engagé pour rendre les milieux marins accessibles aux scientifiques s'est particulièrement fait ressentir sur les côtes françaises avec sa douzaine de stations maritimes, les côtes européennes ont vu pendant la même période s'ériger un certain nombre de stations maritimes (en Italie, Angleterre, Suède, Finlande, Allemagne), dont la Station zoologique de Naples (1872) qui fut « une sorte de prototype d'un centre international indépendant de recherche 
marine et comme une réponse à un besoin largement partagé " $^{8}$. Le fondateur de la station de Naples, Anton Dohrn (1840-1909), fait payer aux États (location d'une ou plusieurs tables) le droit d'envoyer pour quelques mois ou un an, un ou plusieurs scientifiques à la station napolitaine qui, en contrepartie, fournit le local de travail avec le matériel désiré pour la recherche. Cette pratique d'une recherche payante ne plaît pas à tous, en particulier à H. Lacaze-Duthiers qui défend une recherche gratuite pour tous. Aussi, H. Lacaze-Duthiers, l'un des pionniers de la biologie marine, œuvra-t-il dans ce sens quand il fonde les deux premières grandes stations françaises à Roscoff en 1872 et à Banyuls-sur-Mer en 1881. Les chercheurs, qu'ils soient français ou étrangers, avaient dans ces stations gratuitement accès aux locaux et à tout l'outillage nécessaire pour effectuer en toute liberté leur recherche : c'est H. Lacaze-Duthiers qui insiste sur cette conception de la recherche pour souligner toute la différence entre Naples et les deux stations françaises Roscoff et Banyuls.

Ces deux stations sont modestes à l'origine, comme toutes les stations à leur début. $\mathrm{H}$. Lacaze-Duthiers découvre Roscoff en 1868. Il est impressionné par l'amplitude des marées qui offre un découvert de surface exploitable « immense » et « inépuisable ». En face de Roscoff, l'île de Batz protège la côte de la forte mer formant un canal protecteur dans lequel on trouve faune et flore à profusion. Une première maison est louée, puis un bâtiment est acheté, avec d'autres annexes. On aménage, on agrandit ce qui va devenir la station biologique de Roscoff, qui acquiert très vite une réputation internationale. En 1872, 6 à 8 scientifiques pouvaient travailler à Roscoff ; en août 1887, ils sont 25 chercheurs dont 15 sont logés au laboratoire, ce qui était alors une innovation (aujourd'hui, les personnels permanents dépassent la centaine).

La multiplicité des stations maritimes

Les possibilités de travail à Roscoff ne sont guère favorables en hiver, d'où l'idée de $\mathrm{H}$. Lacaze-Duthiers de fonder une nouvelle station dans le Midi. La Méditerranée offre une faune et une flore différentes de celles de la Manche, l'absence de marée conduit à d'autres méthodologies de travail, de récoltes. Banyuls est choisi. Le laboratoire est construit et aménagé pour une grande part sur des fonds privés. Un premier bâtiment est élevé, un aquarium installé, et les bâtiments sont agrandis en plusieurs étapes, avec l'aménagement de chambres après la construction d'une annexe en 1894. Roscoff et Banyuls se complètent dans leurs activités de recherche et d'enseignement. La zoologie dite expérimentale se développe et conduit à des découvertes inattendues, que ce soit dans les formes successives qui conduisent le pentacrine à la comatule, ou la découverte des moyens employés par la sacculine pour parasiter les crabes. Cette dernière fit l'objet de trois années successives d'observations et d'expérimentations de la part d'Y. Delage (1854-1920). On peut citer aussi la découverte par H. Lacaze-Duthiers des mâles pygmées ( 1 à $2 \mathrm{~mm}$ ) portés en parasites par la femelle géante de la bonellie (plus de $30 \mathrm{~cm}$ ) : c'est le triomphe, pour $\mathrm{H}$. Lacaze-Duthiers, d'une zoologie pure et expérimentale. Après qu'il a démonté les mécanismes de la vie des pérophores et des clavelines pour comprendre le fonctionnement de ces animaux vivant en colonies, reliés, attachés, prisonniers les uns des autres, le monde de la mer conduisit le zoologiste à cette réflexion : "Les utopistes qui songent à faire de notre société une association, où la communauté de tout serait la règle universelle, savaient-ils, quand ils élaboraient leurs projets, que la nature les avait devancés dans des proportions bien autrement étendues et positives que tout ce qu'ils avaient rêvé $!^{9}$ " Aussi est-ce sur le terrain que l'enseignement devint particulièrement formateur pour le futur naturaliste : «Un mois passé au bord de la mer, par exemple, lui apprendra davantage qu'une 
année dans le plus riche des musées ou la bibliothèque la mieux pourvue d'ouvrages de zoologie marine ${ }^{10}$.»

Les stations maritimes étaient pour la plupart attachées à une université ou à une autre institution d'État : par exemple, Concarneau au Collège de France, Roscoff et Banyuls à la Sorbonne, Wimereux à l'université de Lille, Sète à celle de Montpellier, Tatihou au Muséum national d'histoire naturelle. Ces stations, dont les vocations premières sont la recherche fondamentale et l'enseignement, collaborent parfois avec les institutions qui organisent et étudient les pêches : Wimereux collabore avec Boulogne-sur-Mer concernant l'étude du plancton qui conditionne l'évolution des bancs de sardines ; Concarneau fait des observations régulières sur les bancs de sardines. Mais le plancton est aussi un matériel de recherche fondamentale et une station comme Villefranche s'est spécialisée dans ce type de recherche.

L'unité des stations maritimes

Chaque station aura une particularité disciplinaire (physiologie, systématique, cytologie, développement...) mais elle est aussi un complément des stations voisines comme lointaines. En raison de l'enseignement diffusé au sein des stations maritimes et de leur ouverture internationale, elles contribuent à un formidable brassage d'hommes et de femmes qui ont, au hasard des rencontres, noué des amitiés et projeté des programmes de recherche conduisant à de fructueuses collaborations.

Un point relie ces institutions : la nécessaire connaissance de cette prodigieuse diversité du monde végétal et animal, modèle d'une vie active, riche d'enseignements qui n'est pas sans nous instruire sur notre condition d'homme. L'ensemble de ces institutions forme un tout qui contribue à la connaissance du monde de la mer et du littoral. Cette connaissance nous a permis de prendre conscience d'une nécessaire protection de cet environnement encore si riche à la fin du XIX ${ }^{e}$ siècle et qui faisait l'émerveillement des pionniers de la biologie.

Les stations maritimes aujourd'hui et le CNRS

17 Le CNRS a contribué et contribue largement à la recherche scientifique ayant un rapport avec la mer et les milieux marins et côtiers par des soutiens financiers et des apports en personnels. Cette participation est encore plus active depuis la création, en 1985 au CNRS, sous les tutelles du ministère de l'éducation nationale, du ministère de la Recherche, de l'Institut national des sciences de l'univers. Celui-ci offre en effet la possibilité de développer des programmes pluridisciplinaires de recherche au niveau national et international.

Citons quelques exemples parmi d'autres de la participation du CNRS à la vie des stations maritimes françaises : en 1946, le CNRS apporte à la station biologique de Roscoff (SBR) un soutien financier de 1,9 million de francs auquel s'ajoutaient 18000 dollars américains de la fondation Rockefeller ${ }^{11}$; en 1945, Georges Teissier (1900-1972), pionnier du développement de la génétique en France et directeur général du CNRS de 1946 à 1950, devient directeur de la SBR (jusqu'en 1971). Depuis mars 1985, la SBR constitue l'école interne numéro 37 de l'université Pierre et Marie-Curie (Paris VI) et possède le statut d'Observatoire océanologique de l'Insu. Deux unités mixtes de recherche (UMR) associées au CNRS regroupent les équipes de recherche de la SBR. Les deux grands axes de recherche sont répartis dans deux départements : $1^{\circ}$ modèles biologiques marins et $2^{\circ}$ adaptation et diversité en milieu marin. Roscoff est l'exemple du fonctionnement d'une station maritime destinée à l'enseignement, à la recherche et à la fourniture de modèles biologiques ; elle est ouverte aux scientifiques nationaux, 
étrangers et aux étudiants ; elle s'ouvre aussi au public avec l'installation d'un aquarium. La station a également en charge une publication scientifique internationale destinée à diffuser les résultats des recherches : les Cahiers de biologie marine, depuis 1960. La station de Roscoff ne fait que poursuivre les objectifs de son fondateur H. Lacaze-Duthiers.

Le modèle de Roscoff se retrouve à l'observatoire océanologique de Villefranche-surMer $^{12}$. Villefranche se caractérise par sa pluridisciplinarité dans les grands thèmes de recherche abordés : biologie cellulaire, océanographie biologique et biochimique, physique et chimie marines, géosciences marines. Le Centre d'océanologie de Marseille (COM) est aussi un observatoire de l'Insu ; il est également membre du Réseau national des stations marines ${ }^{13}$. Les unités mixtes de recherche du COM se répartissent de la façon suivante $: 1^{\circ}$ Laboratoire d'océanologie et biogéochimie, $2^{\circ}$ Diversité biologique et fonctionnement des écosystèmes marins côtiers et $3^{\circ}$ Laboratoire de microbiologie marine.

20 Le CNRS est aussi présent dans des stations de moindre importance (en surface de locaux et en nombre de personnels) comme celle de Wimereux qui se rattache à l'institution sous le nom d'Elico (Écosystèmes littoraux et côtiers). Un autre exemple, celui du Centre de recherche sur les écosystèmes marins et aquacoles de l'Houmeau (près de La Rochelle) de création récente, 1983, est co-géré par le CNRS et l'Ifremer. L'activité du Crema s'articule autour de trois programmes concernant l'écologie côtière $: 1^{\circ}$ couplage pélagos-benthos et fonctionnement des écosystèmes littoraux, $2^{\circ}$ structuration et productivité des communautés microbiennes planctoniques dans les panaches fluviaux et $3^{\circ}$ espèces exploitées dans leurs écosystèmes : réponses adaptatives, interactions, intégration.

21 Si l'Ifremer gère la flotte océanographique hauturière au bénéfice de l'ensemble de la communauté nationale, l'Insu-CNRS gère l'utilisation de la flotte côtière au profit de la communauté scientifique du CNRS et des universités ${ }^{14}$. Ainsi, le CNRS se trouve particulièrement impliqué dans le développement des stations maritimes, aussi bien en bâtiments, matériel et personnels qu'en financements de programmes de recherche nationaux et internationaux.

Parmi les implications du CNRS dans la recherche océanologique, on ne saurait oublier sa participation aux activités de l'Institut universitaire européen de la mer (IUEM), avec le financement de plusieurs unités mixtes de recherche développant des programmes nationaux, européens et internationaux dans les géosciences, l'océanographie biologique, la télédétection, la géomatique et la chimie marine. L'IUEM, situé à Brest, appartient au réseau national des stations marines depuis le 18 décembre 2000 : c'est une fédération de recherche du CNRS. Le CNRS participe aussi aux recherches concernant les sources hydrothermales des abysses et de leur environnement faunique. La dernière expédition (CNRS/Ifremer) dans ce domaine s'est effectuée au large du Mexique du 30 avril au 3 juin 2002. Une partie des récoltes font l'objet d'étude dans des stations maritimes.

Les recherches dans les stations maritimes aujourd'hui

24 S'il fut un temps où la pluridisciplinarité dans les institutions de recherche n'était pas de mise, la pluridisciplinarité qui s'exerce dans les stations maritimes d'aujourd'hui doit être regardée comme un garant de leur pérennité dans la mesure où leur situation géographique et leur confrontation à des milieux divers leur imposent une large palette d'activités de recherche dans des domaines très variés. Aussi l'intérêt de ces 
institutions de la mer et du littoral se trouve-t-il dans la diversité de leurs programmes de recherche qui s'exerce à l'échelle nationale, européenne et internationale.

Le guide du réseau national des stations maritimes a établi une liste de 24 thématiques principales développées dans les stations maritimes. Ces thématiques, sans toutes les nommer, concernent la biologie moléculaire et cellulaire, la biochimie et biophysique, la physiologie, la biodiversité, l'éthologie, les écosystèmes, la biogéochimie, la chimie du milieu marin, la géologie, les biotechnologies, l'aquaculture, la pollution... à la station de Marseille (Endoume), 23 de ces thématiques sont représentées, 21 à Villefranche, 20 à Banyuls, 16 à Wimereux, 15 à l'Houmeau (La Rochelle) et à Luc-surMer, 12 à Roscoff, 11 à Arcachon, 8 à Concarneau... Certaines stations poursuivent des thématiques qui ont fait leur spécificité à une certaine période. Par exemple, à Roscoff, la biologie du développement (cycle cellulaire et développement) a été introduite par son deuxième directeur Y. Delage qui participait dans cette période du début du $\mathrm{XX}^{\mathrm{e}}$ siècle au passionnant débat concernant les mécanismes de la fécondation; il devait se résoudre par la voie expérimentale, et en particulier par la parthénogenèse expérimentale, processus qui consistait, à la suite d'une action chimique, physique ou mécanique sur un ovule, à le conduire à entamer une division cellulaire et une embryogenèse. L'artifice expérimental devait aider à la compréhension de la fécondation par l'action du spermatozoïde qui, selon une réflexion de l'époque, empêche l'ovule de mourir ou lui permet de continuer de vivre. Aujourd'hui, à Roscoff, des équipes poursuivent des recherches sur la fécondation de l'œuf d'oursin pour décrypter les mécanismes moléculaires de la division cellulaire, première étape de l'œuf qui va le conduire vers l'embryogenèse. De même, à Villefranche, un important groupe travaille à la recherche et à la compréhension des mécanismes de la fécondation et du développement précoce. D'autres stations ont une équipe qui consacre son activité à la biologie du développement ; à la station de Banyuls, l'équipe " cycle cellulaire et développement précoce " travaille sur les mécanismes moléculaires de la régulation du cycle de division cellulaire dans la mesure où « la division cellulaire est le moyen universel de propagation des espèces et de croissance des individus ». Ce point commun de recherche fondamentale en biologie du développement, qui réunit certaines stations maritimes dans leur programme de recherche, conduit aussi à montrer un autre intérêt des recherches dans les stations maritimes autour d'un thème fédérateur qui est celui de la pollution (pollution d'origine naturelle et anthropique) et de ses effets sur l'environnement et sur les écosystèmes avec, à la source, ses impacts sur les mécanismes de la fécondation et de la division cellulaire modélisée par l'œuf et la formation de ses premiers blastomères. Il est important de mieux connaître les mécanismes de la division de la cellule car ce sont ces dérèglements qui conduisent à l'appauvrissement d'une espèce ou à sa disparition dans un milieu (le dérèglement de la division de l'œuf entraîne une létalité embryonnaire). Le dérèglement de la division cellulaire est à l'origine de certaines pathologies, comme le cancer. Les résultats de ces études ayant pour modèle l'œuf et l'embryon d'invertébrés marins (oursins, étoiles de mer) sont transposables aux cellules des vertébrés et à l'homme dans la mesure où ses mécanismes sont identiques chez les organismes vivants composés de cellules à noyaux.

Il n'est pas sans intérêt de souligner que souffle encore un esprit naturaliste, qui prend en compte l'intérêt et l'importance de la biodiversité des espèces, sur ces activités de recherche qui animent les stations maritimes, faisant souvent appel à de hautes technologies, ce qui n'est pas une généralité dans les pratiques des sciences de la vie et 
en particulier en biologie moléculaire laquelle, s'attachant à une biodiversité moléculaire, se contente d'un nombre réduit de modèles animaux et végétaux, pouvant rendre, en quelque sorte, obsolète le concept même de biodiversité à l'échelle des espèces et de leurs variétés : ce qui peut alors correspondre à une logique de pensée ne correspond pas forcément à une logique du vivant. Aussi, quand des stations maritimes s'attachent dans leur programme de recherche à travailler sur la dynamique des populations (par exemple à Wimereux), cela implique des connaissances approfondies des milieux et des faunes. Il est important de connaître aussi bien les mécanismes moléculaires de la division cellulaire que les faunes et les flores, de découvrir de nouvelles espèces et de les étudier dans leurs formes, leurs fonctions et leurs comportements. Les grandes disciplines naturalistes et de biologie moléculaire sont réunies dans les stations maritimes. La découverte du monde marin est loin d'être achevée et possède un capital de connaissances auquel le chercheur n'a pas encore eu accès.

27 À Concarneau, on retrouve les disciplines naturalistes, côtoyant celles de la biologie moléculaire et des études ouvertes sur l'économie de la pêche. La station fondée par Coste travaille aujourd'hui à la connaissance de la biologie des poissons, à celle de l'écologie littorale en collaboration avec l'Association pour la découverte du monde sous-marin, à celle des rythmes biologiques des organismes marins et à celle des hormones des crustacés qui s'appuie sur la biologie moléculaire et le génie génétique. Enfin, la station est active dans le domaine des biotechnologies marines. La pluridisciplinarité est là comme dans les autres stations une pratique nécessaire et productive, comme le souligne Yves Le Gal : « De l'établissement du vivier laboratoire en 1859 à nos jours, il y a donc une continuité dans l'évolution des recherches scientifiques à Concarneau. L'objectif de connaissances fondamentales est toujours complété par le souci de répondre, chaque fois que cela est possible, aux préoccupations immédiates de la collectivité, de la gestion du milieu marin à la biologie médicale. Une complémentarité qui est gage d'efficacité et de progrès scientifique. »

En raison de l'intérêt des scientifiques pour l'étude des milieux marins et côtiers et du rôle fondamental que ces milieux jouent sur un équilibre général de la biosphère, les stations maritimes ont un avenir. Celui-ci fut l'objet de réflexions de la part de François Gros en 1994 : « Nous avons vu l'importance du rôle joué par les stations marines dans l'exploration de la biodiversité marine, de la physiologie et de l'écophysiologie et combien l'essor de certaines disciplines - telles la biologie du développement et les neurosciences - a été, dès le début du $\mathrm{XX}^{\mathrm{e}}$ siècle, lié à celui des stations marines. On assiste, d'ailleurs, à une relance de l'intérêt pour les modèles marins dans des recherches aussi diverses que celles sur les facteurs de croissance, la communication inter-organismes, la neurobiologie, l'étude des cycles de division, etc., sans oublier l'intérêt que peut présenter la préservation des organismes marins tant pour le respect des équilibres écologiques que pour des motifs économiques. ${ }^{15}$ " Quoi qu'il en soit, la biologie marine, comme un certain nombre de disciplines ayant un rapport avec la mer, ne peut se situer et se comprendre dans le champ général d'une recherche scientifique qui fonctionne et produit que si nous faisons référence aux Milne-Edwards, Audouin, Coste, van Beneden, Lacaze-Duthiers, Dohrn, Giard ou Dubois qui ont, avec bien d'autres, fondé les sciences de la mer et les institutions pour qu'elles s'y développent. Note de l'auteur 
$29 \mathrm{Au}$ sein du Centre Alexandre-Koyré (CNRS, EHESS, MNHN) existe un groupe de recherche concernant l'histoire des stations maritimes et de la biologie marine. Ce groupe composé de 18 personnes travaille à l'écriture d'une Histoire des stations marines et de la biologie marine (1820-1940) et à celle d'un dictionnaire biographique des stations maritimes. Ce groupe est particulièrement attentif à la recherche, à la conservation et à l'exploitation des archives touchant à l'histoire des stations maritimes. Il est pluridisciplinaire et reflète l'approche historique des stations maritimes qui sont des institutions de recherche fondamentale et appliquée, d'enseignement universitaire et de muséologie.

\section{NOTES}

1.L'adjectif « maritime » (du latin maritimus) qualifie un lieu, une chose « qui est proche de la mer » : une ville maritime, des plantes maritimes et des stations maritimes. Pour qualifier ce « qui appartient à la mer, qui est formé par la mer », nous employons l'adjectif « marin, marine » (du latin marimus) : un monstre marin, des plantes marines, la biologie marine. Si en anglais on écrit marine stations, il n'existe pas de règle imposant la traduction littérale.

2.C. Groeben, « Le précurseur du plan : la contribution de Carl Vogt à la fondation des stations maritimes ", in Carl Vogt, science, philosophie et politique (1817-1895), actes du colloque de mai 1995, édités par J.-C. Pont, D. Bui, F. Dubosson et J. Lacki, éditions Georg, Chêne-Bourg (Suisse), 1998, p. 287-313.

3.H. Lacaze-Duthiers, « Les laboratoires maritimes », Revue scientifique, t. 25, $n^{\circ}$ 7, 18 août 1888, p. 198-212.

4.R. Legendre, « Le rôle des laboratoires maritimes ", Revue scientifique, $\mathrm{n}^{\circ}$ 24, t. 70, 24 décembre 1932, p. 750-753.

5.A. de Quatrefages, Souvenirs d'un naturaliste, Paris, Librairie de Victor Masson, 1854. 6.Les stations maritimes fondées à la fin du XIX ${ }^{\mathrm{e}}$ siècle sont les suivantes : Concarneau (1859), Roscoff (1872), Wimereux (1874), Luc-sur-Mer (1874), Cette (Sète) (1879), Banyuls-sur-Mer (1881), Arcachon (1883), Villefranche-sur-Mer (1886), Tatihou (1887), Le Portel (1888), Endoume (1889), Tamaris (1889-1900), Monaco (1910). À ces stations nous devons ajouter des laboratoires à vocation maritime (Le Havre), des stations discrètes (Pen Château), des stations privées (Embleteuse) et les stations d'outre-mer (Indochine, Madagascar, Algérie, Tunisie...). Concernant l'histoire des stations maritimes, on peut consulter avec intérêt, Kofoid C. A., The Biological Stations of Europe, Washington, Government Printing Office, 1910, 361 p.

7.J. Carpine-Lancre, 1998.

8.C. Groeben, « Le précurseur du plan... », op. cit.

9.H. Lacaze-Duthiers, «Le monde de la mer et ses laboratoires », Revue scientifique, t. 25, $n^{\circ} 6,11$ août 1888, p.162-173.

10.É. Yung, « Une excursion zoologique aux environs de Banyuls-sur-Mer », Revue scientifique, t. $47, \mathrm{n}^{\circ} 22,30$ mai 1891, p. 673-680. 
11.J.-F. Picard, La République des savants, la recherche française et le CNRS, préface de Antoine Prost, Paris, Flammarion, 1990.

12.INSU, École interne $n^{\circ} 33$ de l'université Pierre et Marie-Curie, UMR 6526 CNRS/ UPMC/UNSA/IRD.

13.Le Réseau national des stations marines peut être consulté sur le Web à l'adresse suivante : http://www.sb-roscoff.fr/RNSM/rnsm.html

14.Jean-François Pavillon, Annuaire des chercheurs en sciences de l'eau (limnologues et océanographes), ouvrage réalisé grâce au soutien du ministère de la Recherche, du CNRSINSU, de l'Ifremer et la collaboration de l'Association de l'union des océanographes de France, Paris, Édition de l'Union des océanographes de France, 1998.

15.F. Gros, « Les disciplines marines : situation des stations marines », rapport de l'Académie des sciences, La vie des sciences, Comptes rendus, série générale, t. $11 \mathrm{n}^{\circ} 1$, 1994 , p. 51.

INDEX

Mots-clés : stations maritimes, Roscoff, Concarneau, musée océanographique de Monaco, côte, biologie marine, océanographie, océanographe

\section{AUTEUR}

JEAN-LOUIS FISCHER

Jean-Louis Fischer, chargé de recherche (CNRS), est membre du Centre AlexandreKoyré. 